\title{
The Monodromy Conjecture for hyperplane arrangements
}

\author{
Nero Budur · Mircea Mustaţă • Zach Teitler
}

Received: 2 February 2010 / Accepted: 20 December 2010 / Published online: 6 January 2011

(C) Springer Science+Business Media B.V. 2011

\begin{abstract}
The Monodromy Conjecture asserts that if $c$ is a pole of the local topological zeta function of a hypersurface, then $\exp (2 \pi i c)$ is an eigenvalue of the monodromy on the cohomology of the Milnor fiber. A stronger version of the conjecture asserts that every such $c$ is a root of the Bernstein-Sato polynomial of the hypersurface. In this note we prove the weak version of the conjecture for hyperplane arrangements. Furthermore, we reduce the strong version to the following conjecture: $-n / d$ is always a root of the Bernstein-Sato polynomial of an indecomposable essential central hyperplane arrangement of $d$ hyperplanes in $\mathbf{C}^{n}$.
\end{abstract}

Keywords Monodromy Conjecture $\cdot$ Hyperplane arrangements

Mathematics Subject Classification (2000) $\quad 32 \mathrm{~S} 40 \cdot 32 \mathrm{~S} 22$

\section{Introduction}

Let $f \in \mathbf{C}\left[x_{1}, \ldots, x_{n}\right]$ be a non-constant polynomial, with $f(0)=0$. Consider a $\log$ resolution $\mu: Y \rightarrow \mathbf{C}^{n}$ of $f^{-1}(0)$, and let $E_{i}$ for $i \in S$ be the irreducible components of

The first author was partially supported by the NSF grant DMS-0700360, and the second author was partially supported by NSF grant DMS-0758454 and by a Packard Fellowship.

N. Budur $(\varangle)$

Department of Mathematics, University of Notre Dame, 255 Hurley Hall, Notre Dame, IN 46556, USA

e-mail: nbudur@nd.edu

M. Mustaţă

Department of Mathematics, University of Michigan, 2844 East Hall, 530 Church Street, Ann Arbor, MI 48109-1043, USA

e-mail: mmustata@umich.edu

Z. Teitler

Department of Mathematics, Boise State University, 1910 University Drive, Boise, ID 83725-1555, USA

e-mail: zteitler@boisestate.edu 
$E=(f \circ \mu)^{-1}(0)$. Denote by $a_{i}$ the order of vanishing of $f \circ \mu$ along $E_{i}$, and by $k_{i}$ the order of vanishing of $\operatorname{det}(\operatorname{Jac}(\mu))$ along $E_{i}$. The local topological zeta function of $f$ is

$$
Z_{\mathrm{top}, f}(s):=\sum_{I \subseteq S} \chi\left(E_{I}^{\circ} \cap \mu^{-1}(0)\right) \cdot \prod_{i \in I} \frac{1}{a_{i} s+k_{i}+1},
$$

where $E_{I}^{\circ}=\left(\cap_{i \in I} E_{i}\right)-\cup_{i \notin I} E_{i}$, and $\chi$ is the Euler-Poincaré characteristic. J. Denef and F. Loeser showed in [7] that $Z_{\mathrm{top}, f}(s)$ is independent of the choice of the log resolution $\mu$. The poles of $Z_{\text {top, } f}(s)$ are among the negative rational numbers $\left\{-\left(k_{i}+1\right) / a_{i} \mid i \in S\right\}$.

The Topological Monodromy Conjecture of [7] is a variant of the original Monodromy Conjecture of J.-i. Igusa. To state it, we fix some notation. For $x \in f^{-1}(0)$, let $M_{f, x}$ denote the Milnor fiber of $f$ at $x$, which is defined as the intersection of $f^{-1}(t)$ with a small ball of radius $\epsilon$ around $x(0<t \ll \epsilon \ll 1)$. As a $C^{\infty}$-manifold, $M_{f, x}$ does not depend on $t$ and $\epsilon$.

The Bernstein-Sato polynomial (also called $b$-function) of $f$ is the non-zero monic polynomial $b_{f}(s) \in \mathbf{C}[s]$ of minimal degree among polynomials $b(s)$ satisfying

$$
b(s) f^{s}=P \bullet f^{s+1}
$$

for some algebraic differential operator $P \in \mathbf{C}\left[x_{1}, \ldots, x_{n}, \frac{\partial}{\partial x_{1}}, \ldots, \frac{\partial}{\partial x_{n}}, s\right]$.

Conjecture 1.1 ([7], Topological Monodromy Conjecture) If $c$ is a pole of $Z_{\mathrm{top}, f}(s)$, then

(a) $\exp (2 \pi i c)$ is an eigenvalue of the monodromy action on $H^{i}\left(M_{f, x}, \mathbf{C}\right)$, for some $i$ and some $x \in f^{-1}(0)$.

(b) $b_{f}(c)=0.1$

It is well-known, by results of Malgrange [12] and Kashiwara [11], that all the roots of the $b$-function are negative rational numbers. Also, the set of numbers $\exp (2 \pi i c)$, when $c$ varies over the roots of the $b$-function of $f$, coincides with the set of eigenvalues of the monodromy action on $H^{i}\left(M_{f, x}, \mathbf{C}\right)$ for some $i$, and some $x \in f^{-1}(0)$. Hence part (b) of the above conjecture implies part (a). The main result of this note is to prove part (a) in the case of hyperplane arrangements, and to reduce part (b) in this case to the following

Conjecture 1.2 If $g \in \mathbf{C}\left[x_{1}, \ldots, x_{m}\right]$ is a polynomial of degree $d$, such that $\left(g^{-1}(0)\right)_{\text {red }}$ is an indecomposable, essential, central hyperplane arrangement, then $-\frac{m}{d}$ is a root of the Bernstein-Sato polynomial $b_{g}(s)$.

The following is our main result.

Theorem 1.3 Let $f \in \mathbf{C}\left[x_{1}, \ldots, x_{n}\right]$ define a hyperplane arrangement in $\mathbf{C}^{n}$.

(a) Conjecture 1.1-(a) holds for $f$.

(b) If Conjecture 1.2 holds (for all $g$ ), then Conjecture 1.1-(b) holds for $f$.

In fact, in part (b) the condition has to be checked only for the set of arrangements $f_{W}$ induced on the so-called dense edges $W$ of the given hyperplane arrangement, see Remark 2.4. Theorem 1.3 (a) implies that if $c$ is a pole of $Z_{\mathrm{top}, f}(s)$ then $b_{f}(c)=0$ or $b_{f}(c-1)=0$, since the roots of $b_{f}(s)$ are in $(-2,0)$ in this case, by [14].

The original Monodromy Conjecture of J.-i. Igusa involves $p$-adic local zeta functions. Theorem 1.3 has an analogous version for $p$-adic local zeta functions, see Theorem 2.8 below.

1 A stronger conjecture is believed to be true, where $x$ is close to 0 , and the local $b$-function at 0 is used. However, in our case this makes no difference as we deal with homogeneous polynomials. 
In the last section we discuss several examples, treating in particular generic arrangements, as well as a large class of arrangements.

We would like to thank W. Veys and U. Walther whose interest prompted us to write down this note, and to M. Saito for useful comments and the remarks about moderate arrangements in the last section.

\section{Proof of theorem 1.3}

Let $V_{j} \subset \mathbf{C}^{n}$ be the irreducible components of the reduced hyperplane arrangement $\mathcal{A}:=$ $\left(f^{-1}(0)\right)_{\text {red }}$. We assume the arrangement to be central, that is, each $V_{j}$ is a linear subspace of codimension one of $\mathbf{C}^{n}$. In this case $f$ is homogeneous. We deal at the end of this section with the non-central case.

The following definitions only depend on $f_{\text {red }}$. The central hyperplane arrangement $\mathcal{A}$ is indecomposable if there is no linear change of coordinates on $\mathbf{C}^{n}$ such that $f$ can be written as the product of two nonconstant polynomials in disjoint sets of variables. An edge of $\mathcal{A}$ is an intersection of hyperplanes $V_{i}$. The arrangement $\mathcal{A}$ is essential if $\{0\}$ is an edge of $\mathcal{A}$. An edge $W \subset \mathbf{C}^{n}$ is called dense if the hyperplane arrangement $\mathcal{A}_{W}$ given by the image of $\cup_{V_{j} \supset W} V_{j}$ in $\mathbf{C}^{n} / W$ is indecomposable. For example, $V_{j}$ is a dense edge for every $j$. Let $S$ be the set of dense edges of $\mathcal{A}$. We take $\mu: Y \rightarrow \mathbf{C}^{n}$ to be obtained by successive blowups, by taking for $d=0,1, \ldots, n-2$ the blowup along the (proper transform of) the union of the dense edges of dimension $d$.

Proposition 2.1 ([15]-Theorem 3.1) The morphism $\mu: Y \rightarrow \mathbf{C}^{n}$ is a log resolution of $f^{-1}(0)$.

This $\log$ resolution is the same as the wonderful model of [5] when the building set is taken to be the minimal one, which is exactly the set of dense edges. However, we do not need this additional description. Let $E_{W} \subset Y$ be the proper transform of the exceptional divisor corresponding to $W \in S$. We have: $k_{W}=\operatorname{codim} W-1$ and $a_{W}=\sum_{W \subseteq V_{j}} m_{j}$ for all $W \in S$, where $a_{W}, k_{W}$ are as in (1), and $m_{j}$ is the order of vanishing of $f$ along $V_{j}$ (hence $m_{j}=1$ if $f$ is reduced).

Lemma 2.2 ([15]-Proposition 2.6) Let $g \in \mathbf{C}\left[x_{1}, \ldots, x_{m}\right]$ be such that $\mathcal{B}=\left(g^{-1}(0)\right)_{\text {red }}$ is a central hyperplane arrangement in $\mathbf{C}^{m}$. If $U=\mathbf{P}^{m-1} \backslash \mathbf{P}(\mathcal{B})$, where $\mathbf{P}(\mathcal{B})$ is the projectivization of $\mathcal{B}$, then $\mathcal{B}$ is indecomposable if and only if $\chi(U) \neq 0$.

Lemma 2.3 If $g \in \mathbf{C}\left[x_{1}, \ldots, x_{m}\right]$ with $\operatorname{deg} g=d$ gives an indecomposable central hyperplane arrangement $\mathcal{B}=\left(g^{-1}(0)\right)_{\text {red }} \subset \mathbf{C}^{m}$, then $\{\exp (2 \pi i k / d) \mid k=1, \ldots, d\}$ is the union of the sets of eigenvalues of the monodromy action on $H^{i}\left(M_{g, 0}, \mathbf{C}\right)$ with $i=0, \ldots, m-1$.

Proof It is known that for every homogeneous polynomial $g$, the Milnor fiber $M_{g, 0}$ is diffeomorphic with $g^{-1}(1)$, and gives a finite $d$ to 1 unramified cover of $U:=\mathbf{P}^{m-1} \backslash \mathbf{P}(\mathcal{B})$ (see $[3$, p. 72] (1.13)). It is also well-known that each eigenvalue of the action of the monodromy on the cohomology of the Milnor fiber is of the form $\exp (2 \pi i k / d)$ (see [2]). Conversely, recall that the monodromy zeta function of $g$ is by definition

$$
\prod_{0 \leq j \leq m-1} \operatorname{det}\left(\operatorname{Id}-t \cdot M_{j}\right)^{(-1)^{j}},
$$

where $M_{j}$ is the monodromy action on $H^{j}\left(M_{g, 0}, \mathbf{C}\right)$. By Example 6.1.10 in [4], the monodromy zeta function of $g$ is $\left(1-t^{d}\right)^{\chi\left(M_{g, 0}\right) / d}$. Let $\chi_{c}(-)$ denote the Euler characteristic with 
compact supports. Since $\chi\left(M_{g, 0}\right)=\chi_{c}\left(M_{g, 0}\right)$ and $\chi(U)=\chi_{c}(U)$ (see for example [9], p. 141 , note 13$)$, it follows from the multiplicativity property of $\chi_{c}(-)$ with respect to finite unramified coverings that $\chi\left(M_{g, 0}\right) / d=\chi(U)$. It follows from Lemma 2.2 that for every $k, \exp (2 \pi i k / d)$ is an eigenvalue of $M_{j}$ on $H^{j}\left(M_{g, 0}, \mathbf{C}^{n}\right)$, for some $j$.

We can now prove the result stated in the Introduction for the case of central arrangements.

Proof of part (a) of Theorem 1.3 By the definition (1) and Proposition 2.1, the poles of $Z_{\text {top, } f}(s)$ are included in the set $\left\{-(\operatorname{codim} W) / a_{W} \mid W \in S\right\}$. If $W \in S$, then $\mathcal{A}_{W}$ is indecomposable and is the reduced zero locus of a product $f_{W}$ of linear forms on $\mathbf{C}^{n} / W$, with $\operatorname{deg}\left(f_{W}\right)=a_{W}$. By Lemma 2.3, $\exp \left(-2 \pi i(\operatorname{codim} W) / a_{W}\right)$ is an eigenvalue of the monodromy on the Milnor cohomology of $f_{W}$ at the origin in $\mathbf{C}^{n} / W$. Now, take a point $p \in W-\cup_{V_{j} \not \supset W} V_{j}$. After choosing a splitting of $W \subset \mathbf{C}^{n}$, we have locally around $p, \mathcal{A}=$ $\mathcal{A}_{W} \times W \subset \mathbf{C}^{n}=\mathbf{C}^{n} / W \times W$ and $f=f_{W} \cdot u$, where $u$ is a (locally) invertible function. Hence the Milnor fiber of $f_{W}$ at the origin is a deformation retract of $M_{f, p}$. Therefore $\exp \left(-2 \pi i(\operatorname{codim} W) / a_{W}\right)$ is an eigenvalue of the monodromy on the cohomology of the Milnor fiber of $f$ at $p$.

Proof of part (b) of Theorem 1.3 We assume that for all $m, d>0$, and for all polynomials $g$ of degree $d$ in $m$ variables defining an indecomposable, essential, central hyperplane arrangement, we have $b_{g}(-m / d)=0$. By Proposition 2.1, the candidate poles of $Z_{\text {top, } f}(s)$ are $-(\operatorname{codim} W) / a_{W}$, with $W \in S$. Let $f_{W}$ be induced by $f$ as before, such that $\mathcal{A}_{W}=\left(f_{W}^{-1}(0)\right)_{\text {red }}$ in $\mathbf{C}^{n} / W$. Then $(\operatorname{codim} W) / a_{W}=\operatorname{dim}\left(\mathbf{C}^{n} / W\right) / \operatorname{deg} f_{W}$, with $\mathcal{A}_{W}$ indecomposable (and automatically essential and central) in $\mathbf{C}^{n} / W$. By assumption, we have $b_{f_{W}}\left(-(\operatorname{codim} W) / a_{W}\right)=0$. On the other hand, $b_{f_{W}}(s)$ equals the local $b$-function $b_{f, p}(s)$ for $p$ as in the proof of part (a). But $b_{f}(s)$ is the least common multiple of the local $b$-functions. Hence every candidate pole as above is a root of $b_{f}(s)$.

Remark 2.4 It follows from the above proof that in order for Conjecture 1.1-(b) to hold for $f$, it is enough to have Conjecture 1.2 satisfied by all $f_{W}$, with $W \in S$.

Remark 2.5 (Non-central arrangements.) Let $f \in \mathbf{C}\left[x_{1}, \ldots, x_{n}\right]$ be such that $\left(f^{-1}(0)\right)_{\text {red }}=$ $\cup_{j} V_{j}$ is a not necessarily central hyperplane arrangement. Let $f_{0}$ be the polynomial obtained from $f$ by only considering the factors that vanish at 0 . In this case $Z_{\text {top, } f}(s)=Z_{\text {top, } f_{0}}(s)$. We showed that the exponentials of the poles of $Z_{\text {top, } f_{0}}(s)$ are included in the union over indecomposables $W$ for $f_{0}$ of the eigenvalues of monodromy on $M_{f_{0}, x}$, where $x$ is any point of $W-\cup_{0 \in V_{j} \not \supset W} V_{j}$. Also, assuming Conjecture 1.2, the poles of $Z_{\text {top, } f_{0}}(s)$ are included in the union over indecomposables $W$ for $f_{0}$ of the roots of $b_{f_{0}, x}$. We can choose the point $x$ such that $x \notin \cup_{0 \notin V_{j}} V_{j}$. In this case $M_{f_{0}, x}=M_{f, x}$ and $b_{f_{0}, x}=b_{f, x}$. This shows that Theorem 1.3 is valid for the case of non-central arrangements as well.

2.6 The $p$-adic case. We next consider the $p$-adic version of the Monodromy Conjecture. For a polynomial $f \in \mathbf{Q}\left[x_{1}, \ldots, x_{n}\right]$ and a prime number $p$, Igusa's $p$-adic local zeta function of $f$ is defined as $Z_{f}^{p}(s)=\int_{\left(\mathbf{Z}_{p}\right)^{n}}|f|_{p}^{s} d \mu$, where $d \mu$ is the Haar measure on $\left(\mathbf{Q}_{p}\right)^{n}$, and $|.|_{p}$ is the $p$-adic norm. $Z_{f}^{p}(s)$ is a meromorphic function for $s \in \mathbf{C}$ whose poles encode asymptotic behavior of the numbers $N_{m}$ of solutions of $f$ modulo $p^{m}$ when $f$ is defined over $\mathbf{Z}$, see [6]. Let $\operatorname{Re}(c)$ denote the real part of a complex number $c$.

Conjecture 2.7 ([10], Monodromy Conjecture) Let $f \in \mathbf{Q}\left[x_{1}, \ldots, x_{n}\right]$. For almost all prime numbers $p$, if $c$ is a pole of $Z_{f}^{p}(s)$, then

(a) $\exp (2 \pi i \cdot \operatorname{Re}(c))$ is an eigenvalue of the monodromy action on $H^{i}\left(M_{f, x}, \mathbf{C}\right)$, for some $i$ and some $x \in \mathbf{C}^{n}$ with $f(x)=0$. 
(b) $b_{f}(\operatorname{Re}(c))=0$.

It is speculated that the conjectures might be true for all prime numbers $p$, see [6]-2.3.

The analog of Theorem 1.3 involves $\mathbf{Q}$-hyperplane arrangements. These are hyperplane arrangements in $\mathbf{C}^{n}$ such that each hyperplane, i.e. irreducible component, is defined over $\mathbf{Q}$. Note that this implies that every edge is defined over $\mathbf{Q}$. Let $f \in \mathbf{Q}\left[x_{1}, \ldots, x_{n}\right]$. We say that $f$ defines a $\mathbf{Q}$-hyperplane arrangement if $f$, viewed as a polynomial with complex coefficients, does. In other words, $f$ splits completely into linear factors defined over $\mathbf{Q}$.

Theorem 2.8 Let $f \in \mathbf{Q}\left[x_{1}, \ldots, x_{n}\right]$ define a $\mathbf{Q}$-hyperplane arrangement.

(a) Conjecture 2.7-(a) holds for $f$ and all prime numbers $p$.

(b) If Conjecture 1.2 holds for all $f_{W}$ where $W$ is a dense edge of $f$, then Conjecture 2.7-(b) holds for $f$ and all prime numbers $p$.

Proof Let $\mu$ be the log resolution of $f$ from Proposition 2.1. Consider the hyperplane arrangement $f^{(p)}$ defined by $f$ in $\left(\mathbf{Q}_{p}\right)^{n}$. Any dense edge of $f^{(p)}$ is also defined over $\mathbf{Q}_{p}$. By taking successive blowing ups of $\left(\mathbf{Q}_{p}\right)^{n}$ along (proper transforms) of dense edges as in Proposition 2.1, the map $\mu^{(p)}$ thus obtained is a $\log$ resolution of $f^{(p)}$ over $\mathbf{Q}_{p}$. There is a bijection between the dense edges of $f$ and those of $f^{(p)}$, and the orders of vanishing of $f \circ \mu$ and $f^{(p)} \circ \mu^{(p)}$ along the irreducible divisors corresponding to dense edges are the same. Similarly for the orders of vanishing of $\operatorname{det}(\operatorname{Jac}(\mu))$ and $\operatorname{det}\left(\operatorname{Jac}\left(\mu^{(p)}\right)\right)$. Then, by [10]-Theorem 8.2.1 (see also bottom of p. 123 of [10]), the real parts of the poles of $Z_{f}^{p}(s)$ are among the set $\left\{-(\operatorname{codim} W) / a_{W} \mid W\right.$ dense edge of $\left.f\right\}$. From now on, the proof is the same as for Theorem 1.3.

Remark 2.9 Conjecture 2.7 was originally stated with $\mathbf{Q}$ replaced by any number field $F \subset \mathbf{C}, \mathbf{Q}_{p}$ replaced by a $p$-adic completion $K$ of $F$, and $Z_{f}^{p}(s)$ replaced by an integral over $\left(\mathcal{O}_{K}\right)^{n}$, see [6]-2.3. We get the corresponding more general version of Theorem 2.8 , and the proof, by considering $F$-hyperplane arrangements and all $p$-adic completions $K$.

\section{Examples}

In this section we discuss some examples for which we can prove Conjecture 1.2. We start with the case of generic hyperplane arrangements. Recall that a central hyperplane arrangement $\mathcal{A}$ is generic if for every nonzero edge $W$, there are precisely codim $(W)$ hyperplanes in $\mathcal{A}$ containing $W$. Such an arrangement is essential if and only if the number of hyperplanes is at least the dimension of the ambient space.

Example 3.1 Let $g \in \mathbf{C}\left[x_{1}, \ldots, x_{n}\right]$ be a reduced polynomial, defining a generic central, essential arrangement of $d$ hyperplanes in $\mathbf{C}^{n}$. It was shown in [17] that the roots of $b_{g}$ are those $-\frac{i+n}{d}$, with $0 \leq i \leq 2 d-n-2$. In particular, we see that for $i=0$ we get that $-\frac{n}{d}$ is a root of $b_{g}$, confirming Conjecture 1.2 in this case. (Note that a generic arrangement is decomposable if and only if $d=n$, when in suitable coordinates the arrangement consists of the coordinate hyperplanes).

Note that every central arrangement in $\mathbf{C}^{2}$ is generic, hence Example 3.1 applies.

Remark 3.2 Let $g \in k[x, y, z]$ be a reduced polynomial, defining a central, essential arrangement of $d$ hyperplanes in $\mathbf{C}^{3}$. In this case Conjecture 1.1-(b) holds for $g$ if Conjecture 1.2 
holds for $g$, that is, if either $\mathcal{A}$ is decomposable, or if $b_{g}(-3 / d)=0$. Indeed, this follows from Remark 2.4, together with the fact that if $W \neq\{0\}$, then $g_{W}$ satisfies Conjecture 1.2 by Example 3.1.

A recent computation, that will appear elsewhere, of M. Saito, S. Yuzvinsky, and the first author, shows that Conjecture 1.2 is true for $m=3$ and $g$ reduced, and hence Conjecture 1.1(b) is also true.

It is known that every jumping number in $(0,1]$ is the negative of a root of the BernsteinSato polynomial, see [8], Theorem B (but not conversely). So to show Conjecture 1.2 it is enough to show $m / d$ is a jumping number. We now consider some cases in which it can be shown $m / d$ is a jumping number, and an example in which it is not.

We first consider reduced polynomials defining central, essential hyperplane arrangements in $\mathbf{C}^{3}$. Let $g \in \mathbf{C}[x, y, z]$ be such a polynomial defining the arrangement $\mathcal{A}$. We denote by $\mathbf{P}(\mathcal{A})$ the corresponding arrangement of lines in $\mathbf{P}^{2}$. For every $m \geq 2$, let $v_{m}$ be the number of points with multiplicity $m$ in $\mathbf{P}(\mathcal{A})$. Note that $\mathcal{A}$ is generic if and only if there are no points in $\mathbf{P}(\mathcal{A})$ of multiplicity $>2$, and in this case Conjecture 1.2 is satisfied by Example 3.1. We also point out that since the arrangement is in $\mathbf{C}^{3}$, it is decomposable if and only if all but one of the lines in $\mathbf{P}(\mathcal{A})$ pass through a point.

Example 3.3 Suppose that $g$ is as in the previous remark. Corollary 1 in [1] implies that $3 / d$ is a jumping number for the multiplier ideals of $g$ if and only if

$$
\sum_{2 d / 3<m \leq d} v_{m} \neq 1
$$

(here we assume for simplicity that $d \geq 5$ ). It follows that whenever (2) holds, Conjecture 1.2 holds for $g$. In particular, using Remark 3.2 and Example 3.1, one can check for example that Conjecture 1.1-(b) holds for all (reduced) central hyperplane arrangements $\mathcal{A}$ in $\mathbf{C}^{3}$ such that the multiplicities at points of $\mathbf{P}(\mathcal{A})$ are $\leq 3$. We will state a stronger result, for moderate arrangements, in Theorem 3.6.

Remark 3.4 Even in the case of hyperplane arrangements defined by a reduced polynomial $g$, in general it is not the case that one can prove Conjecture 1.2 by showing that $m / d$ is a jumping number for the multiplier ideals of $g$. For example, if $g=x y(x-y)(x+y)(x+z)$, then $3 / 5$ is not a jumping number (one can use, for example, Corollary 1 in [1]). However, this arrangement is decomposable, so Conjecture 1.1-(b) holds by Remark 3.2. In order to get an indecomposable example, we consider an arrangement with $d=10, v_{7}=1, v_{3}=3$, and with $v_{m^{\prime}}=0$ for $7 \neq m^{\prime}>3$. In this case we see by loc. cit. that $3 / 10$ is not a jumping number. For example, we may take

$$
g=x y(x+y)(x-y)(x+2 y)(x+3 y)(x+4 y)(2 y+z)(x+2 y+z) z .
$$

On the other hand, it can be checked that $-3 / 10$ is indeed a root of $b_{g}(s)$, as predicted by Conjecture 1.2. For this one uses Theorem 4.2 (e) in [14], with $k=3$, and $I=\{1,2\}$ as a subset of the set $\{1, \ldots, 10\}$ that remembers the factors of $g$ in the order written.

The following remarks were pointed out to us by M. Saito. Let $\mathcal{A}$ be a hyperplane arrangement. For an edge $W$, let $a_{W}$ be, as before, the sum $\sum_{W \subseteq V_{j}} m_{j}$ where $m_{j}$ is the multiplicity of the hyperplane $V_{j}$ in $\mathcal{A}$. We say that $\mathcal{A}$ is a hyperplane arrangement of moderate type if the following condition is satisfied:

$$
\frac{\operatorname{codim} W}{a_{W}} \leq \frac{\operatorname{codim} W^{\prime}}{a_{W^{\prime}}} \text { for any two dense edges } W \subset W^{\prime} .
$$


Lemma 3.5 If $\mathcal{A}$ is a hyperplane arrangement of moderate type, then (codim $W$ )/ $a_{W}$ is a jumping number of $\mathcal{A}$ for any dense edge $W$.

Proof For any given dense edge $W$, we may assume that there is strict inequality in (3) by replacing $W$ with an edge containing it without changing $(\operatorname{codim} W) / a_{W}$. The conclusion follows directly from the formula for multiplier ideals in terms of dense edges of [16].

By [8], Lemma 3.5 implies:

Theorem 3.6 If $\mathcal{A}$ is a hyperplane arrangement of moderate type, then for any dense edge $W,-(\operatorname{codim} W) / a_{W}$ is a root of the $b$-function of a polynomial defining $\mathcal{A}$. So Conjecture 1.2 holds in this case.

Remark 3.7 (i) Lemma 3.5 does not hold if $\mathcal{A}$ is not of moderate type as is shown in Remark 3.4.

(ii) It is also possible to prove Theorem 3.6 by using [14], 4.2(b).

(iii) If $\mathcal{A}$ is central, essential, and reduced, and $n=3$, then condition (3) holds if and only if the multiplicity along any codimension 2 edge is at most $2 d / 3$, where $d$ is the number of hyperplanes of $\mathcal{A}$. Hence Theorem 3.6 strengthens Example 3.3.

\section{References}

1. Budur, N., Saito, M.: Jumping coefficients and spectrum of a hyperplane arrangement. Math. Ann. 347(3), 545-579 (2010)

2. Cohen, D., Suciu, A.: On Milnor fibrations of arrangements. J. London Math. Soc. 51(2), 105-119 (1995)

3. Dimca, A.: Singularities and Topology of Hypersurfaces. Universitext, Springer, New York (1992)

4. Dimca, A.: Sheaves in Topology. Universitext, Springer, Berlin (2004)

5. De Concini, C., Procesi, C.: Wonderful models of subspace arrangements. (Selecta Math. (N.S.) 1(3), 459-494 (1995)

6. Denef J.: Report on Igusa's local zeta function. Séminaire Bourbaki, Vol. 1990/91. Astérisque No. 201-203 (1991), Exp. No. 741, 359-386 (1992)

7. Denef, J., Loeser, F.: Caractéristiques d'Euler-Poincaré, fonctions zéta locales et modifications analytiques. J. Am. Math. Soc. 5(4), 705-720 (1992)

8. Ein, L., Lazarsfeld, R., Smith, K.E., Varolin, D.: Jumping coefficients of multiplier ideals. Duke Math. J. 123, 469-506 (2004)

9. Fulton, W.: Introduction to Toric Varieties, Annals of Mathematics Studies 131. Princeton University Press, Princeton, NJ (1993)

10. Igusa, J.-i.: An introduction to the theory of local zeta functions, AMS/IP Studies in Advanced Mathematics. 14. American Mathematical Society, Providence, RI; International Press, Cambridge, MA (2000)

11. Kashiwara, M.: $B$-functions and holonomic systems. Rationality of roots of $B$-functions. Invent. Math. 38(1), 33-53 (1976/1977)

12. Malgrange, B.:Polynôme de Bernstein-Sato et cohomologie évanescente, Analysis and topology on singular spaces, II, III (Luminy, 1981), Astérisque 101-102 (1983), 243-267

13. Mustaţă, M.: Multiplier ideals of hyperplane arrangements. Trans. Am. Math. Soc. 358(11), 5015$5023(2006)$

14. Saito, M.: Bernstein-Sato polynomials of hyperplane arrangements. math.AG/0602527

15. Schechtman, V., Terao, H., Varchenko, A.: Local systems over complements of hyperplanes and the Kac-Kazhdan conditions for singular vectors. J. Pure Appl. Algebra 100, 93-102 (1995)

16. Teitler, Z.: A note on Mustaţă's computation of multiplier ideals of hyperplane arrangements. Proc. Am. Math. Soc. 136, 1575-1579 (2008)

17. Walther, U.: Bernstein-Sato polynomial versus cohomology of the Milnor fiber for generic hyperplane arrangements. Compos. Math. 141, 121-145 (2005) 